\title{
ALOCAÇÃO DE NUTRIENTES EM PLANTIOS DE EUCALIPTO NO BRASIL ${ }^{(1)}$
}

\author{
Reynaldo Campos Santana ${ }^{(2)}$, Nairam Félix de $\operatorname{Barros}^{(3)}$, Roberto \\ Ferreira Novais $^{(3)}$, Hélio Garcia Leite ${ }^{(4)} \&$ Nicholas Brian Comerford $^{(5)}$
}

\begin{abstract}
RESUMO
Práticas de manejo florestal podem alterar a exportação de nutrientes do sítio. Este trabalho teve por objetivo estimar o conteúdo de nutrientes em árvores de eucalipto, em diferentes regiões do Brasil. Avaliou-se a influência de algumas características climáticas na produção e no conteúdo de nutrientes na biomassa, utilizando-se o banco de dados do Programa de Pesquisa em Solos e Nutrição de Eucalipto do Departamento de Solos - UFV. As características climáticas foram um importante componente dos modelos. A produção de biomassa e o conteúdo de nutrientes foram positivamente relacionados entre si e ambos foram menores nas regiões com menor disponibilidade de água. As estimativas apontaram que até à idade de 4,5 anos pós-plantio acumulam-se as maiores proporções de nutrientes ( $68 \%$ do N, $69 \%$ do $\mathrm{P}, 67 \%$ do $\mathrm{K}, 63 \%$ do $\mathrm{Ca}$ e $68 \%$ do $\mathrm{Mg}$ ) para a idade de corte de 6,5 anos. Isto indica que, após 4,5 anos, o potencial de resposta à aplicação de fertilizantes é menor. $O$ conteúdo estimado de nutrientes acumulados na copa e na casca representou $65,70,64,79$ e $79 \%$, de $\mathrm{N}, \mathrm{P}, \mathrm{K}, \mathrm{Ca}$ e $\mathrm{Mg}$, respectivamente, até 6,5 anos de idade. Assim, a colheita apenas do lenho representa expressiva redução na exportação desses nutrientes proporcionando maior sustentabilidade da produção nas plantações de eucalipto.
\end{abstract}

Termos de indexação: nutrição florestal; biomassa; macronutrientes.

\footnotetext{
(1) Trabalho apresentado no XXXI Congresso Brasileiro de Ciência do Solo, 2007 (Gramado, RS).

(2) Professor do Departamento de Engenharia Florestal da Universidade Federal dos Vales do Jequinhonha e Mucuri-UFVJM. CEP: 39.100-000 Diamantina (MG) E-mail:silviculturaufvjm@yahoo.com.br

(3) Professor do DPS/Universidade Federal de Viçosa-UFV. CEP: 36.571-000 Viçosa (MG), bolsista do CNPq. E-mail: nfbarros@ufv.br e rfnovais@ufv.br

(4) Professor do Departamento de Engenharia Florestal - UFV. Bolsista do CNPq. E-mail: hgleite@ufv.br

(5) Center Director and Professor, North Florida Research and Education Center, University of Florida, 155 Research Road, Quincy, Florida 32353. E-mail: nbc@ufl.edu
} 


\title{
SUMMARY: NUTRIENT ALLOCATION IN EUCALYPT PLANTATIONS IN BRAZIL
}

\begin{abstract}
Forest management practices can alter nutrient exportation from the site. The purpose of this study was to estimate nutrient contents in the aboveground biomass of eucalyptus plantations in Brazil. The influence of key climatic variables on eucalypt productivity and nutrient content was evaluated, using the database from the Reserch Programa on Soil and Eucalyptus Nutrition of the Soil Science, Departament - Federal University of Viçosa, Minas Gerais State, Brazil. Climatic characteristics were an important component of the models. In regions with low water availability the nutrient accumulation in aboveground biomass as well as biomass production were lower. The highest proportion of all nutrients (68\% N, $69 \% \mathrm{P}, 67 \% \mathrm{~K}, 63 \% \mathrm{Ca}$, and $68 \% \mathrm{Mg}$ ) was accumulated in the biomass within 4.5 years after planting for harvesting after approximately 6.5 years. After 4.5 years, the potential of fertilizer response was lower. Canopy and stem bark together accounted for $65 \% \mathrm{~N}, 70 \% \mathrm{P}, 64 \% \mathrm{~K}, 79 \% \mathrm{Ca}$, and 79 $\% \mathrm{Mg}$ in the aboveground biomass. Debarking in the field can therefore substantially reduce nutrient exportation and contribute to a more sustainable production in eucalyptus plantations.
\end{abstract}

Index terms: Forest nutrition; biomass; macronutrients.

\section{INTRODUÇÃO}

Nos últimos anos, o plantio de eucalipto no Brasil expandiu-se para regiões além daquelas tradicionais, como a Sul e Sudeste, tornando necessária a obtenção de informações sobre a produção e demanda de nutrientes desses novos plantios.

A produção de uma floresta é determinada pela quantidade de radiação solar interceptada pela copa e pela eficiência de conversão da radiação em biomassa. Essa eficiência é principalmente influenciada pela disponibilidade de água e de nutrientes. Aumentando o suprimento destes fatores, além do ponto de deficiência, tende a aumentar a produção (Epstein \& Bloom, 2006) que é fortemente influenciada pelas interações entre eles até que seja atingido um nível ótimo.

Os fatores de crescimento podem tornar-se limitantes para as plantas por poucos minutos (temperatura); por dias, semanas (água) ou até mesmo meses (nutrientes). A radiação solar e a concentração de $\mathrm{CO}_{2}$ da atmosfera são fatores não-manejáveis em condições normais e tendem a não limitar o crescimento nos trópicos. Nestas condições, a água e os nutrientes são os principais limitantes da produtividade florestal (Barros \& Comerford, 2002) reduzindo-a em relação ao potencial máximo permitido pela disponibilidade de radiação (Linder et al., 1996).

A sustentabilidade da produtividade de uma floresta é determinada, dentre outros fatores, pelo balanço de nutrientes no sistema solo-planta. Evidências da interação entre água e nutrientes influenciando a produção de biomassa têm sido obtidas em experimentos de longa duração com Pinus radiata na
Austrália (Raison et al., 1992) e com E. globulus em Portugal (Pereira et al., 1994) e respostas positivas à adição de diferentes nutrientes e, ou, às suas interações com a água têm sido relatadas para plantios de eucalipto no Brasil (Barros \& Comerford, 2002; Stape, et al., 2006). A produção de biomassa é também determinada pelas características genéticas da espécie e maiores produtividades estão associadas com maiores exportações de nutrientes do sítio (Santana et al., 2002).

Para adoção de estratégias adequadas de manejo a longo prazo, os silvicultores devem conhecer as relações entre a quantidade de nutrientes que é exportada e a biodisponibilidade dos nutrientes no sitio de cultivo, para poder aplicar técnicas que visem ao manejo florestal sustentável por várias rotações.

Este estudo visou a avaliar a biomassa, o conteúdo e a alocação de nutrientes em árvores de eucalipto em diferentes idades e regiões do Brasil.

\section{MATERIAL E MÉTODOS}

O Departamento de Solos da Universidade Federal de Viçosa, entre os anos de 1981 e 2000, formou um banco de dados sobre a produção de biomassa e conteúdo de nutrientes nela contidos em plantios de eucalipto cultivados em várias condições edafoclimáticas brasileiras. As espécies e os clones de eucalipto utilizados constituíam o melhor material genético disponível na época da amostragem para cada região. As práticas de manejo florestal podem ter variado, mas representaram o que melhor se conhecia na época. Em geral, o preparo do solo foi realizado por 
grade bedding ou arado de disco, nas linhas de plantio. A fertilização foi feita em faixa, sulco ou cova na época de plantio e em cobertura, aos 18 meses de idade.

A biomassa e o conteúdo de nutrientes foram determinados em povoamentos de seis meses a 11 anos de idade, em parcelas $\left(600 \mathrm{~m}^{2}\right)$ distribuídas aleatoriamente e representativas de cada região. Todas as árvores das parcelas tiveram seu diâmetro (a 1,3 m de altura do solo) e sua altura comercial medidos. Neste trabalho, foram mensuradas 1.217 árvores-amostras, em 20 regiões de cinco Estados (Quadro 1). Em cada parcela, uma árvore média (árvore-amostra) foi abatida e seus componentes (folha, galho, casca e lenho) foram pesados no campo. Subamostras foram coletadas e secas a $65^{\circ} \mathrm{C}$ em estufa com circulação forçada de ar. Após a determinação da matéria seca dos componentes das árvores (folha, galho, casca e lenho), as amostras do material vegetal foram moídas, passadas por peneiras e submetidas à digestão nítricoperclórica; as concentrações de $\mathrm{P}$ (colorimetria), K (fotometria de emissão de chama), Ca e $\mathrm{Mg}$ (espectrometria de absorção atômica) foram determinadas no extrato, enquanto o $\mathrm{N}$ foi determinado pelo método de Kjeldahl.

A biomassa e o conteúdo dos nutrientes dos componentes das árvores, por hectare, foram obtidos ao multiplicar a matéria seca e o conteúdo de nutrientes de cada componente das árvores-amostra pelo número de árvores em um hectare. A estimativa de produção de biomassa e do conteúdo de nutrientes da parte aérea em diferentes regiões foi obtida conforme proposto por Santana (2000).

Quadro 1. Material genético, número de árvores-amostra e valores médios de características climáticas utilizadas no ajuste das equações de produção de biomassa e conteúdos de nutrientes da parte aérea, em eucalipto cultivado em diversas regiões do Brasil

\begin{tabular}{|c|c|c|c|c|c|c|c|}
\hline \multirow{2}{*}{ Região } & \multirow{2}{*}{$\begin{array}{l}\text { Material } \\
\text { genético* }\end{array}$} & \multirow[t]{2}{*}{$n$} & \multicolumn{5}{|c|}{ Característica climática } \\
\hline & & & $P$ & PMS & $E V P$ & $W$ & $T$ \\
\hline & & & \multicolumn{4}{|c|}{$-\mathrm{mm}$} & ${ }^{\circ} \mathrm{C}$ \\
\hline Três Marias - MG & $3,4,5,6,7,8,9$ & 177 & 1.280 & 240 & 1.320 & -40 & 23 \\
\hline Lençóis Paulista - SP & 1 & 35 & 1.200 & 240 & 1.200 & 0 & 20 \\
\hline Luiz Antônio - SP & $1,2,3,5$ & 207 & 1.440 & 240 & 1.380 & 60 & 20 \\
\hline Carbonita - MG & 1 & 12 & 1.280 & 240 & 1.260 & 20 & 22 \\
\hline Itamarandiba $-\mathrm{MG}$ & 10 & 23 & 1.280 & 240 & 1.260 & 20 & 22 \\
\hline Bom Despacho - MG & 1 & 13 & 1.440 & 240 & 1.260 & 180 & 21 \\
\hline Paraopeba - MG & 1 & 11 & 1.440 & 240 & 1.260 & 180 & 21 \\
\hline Itapetininga - SP & 1,2 & 13 & 1.280 & 320 & 1.080 & 200 & 20 \\
\hline Angatuba - SP & 1,2 & 16 & 1.280 & 320 & 1.080 & 200 & 20 \\
\hline Depressão Central - RS & 2 & 53 & 1.440 & 720 & 780 & 660 & 14 \\
\hline Guanhães - MG & 1 & 40 & 1.280 & 240 & 1.080 & 200 & 22 \\
\hline Viçosa - MG & 1 & 11 & 1.360 & 240 & 900 & 460 & 21 \\
\hline Timóteo - MG & $1,4,10$ & 36 & 1.520 & 320 & 1.080 & 440 & 21 \\
\hline São Miguel Arcanjo - SP & 1,2 & 12 & 1.440 & 480 & 1.080 & 360 & 20 \\
\hline Itapeva - SP & 1 & 18 & 1.440 & 480 & 1.080 & 360 & 20 \\
\hline Ipatinga - MG & 1,5 & 20 & 1.520 & 320 & 1.080 & 440 & 22 \\
\hline Litoral Norte - ES & 1,3 & 249 & 1.200 & 320 & 1.080 & 120 & 24 \\
\hline São José dos Campos - SP & 1,2 & 34 & 1.840 & 480 & 600 & 1.240 & 19 \\
\hline Paraibuna - SP & 1,2 & 18 & 1.840 & 480 & 540 & 1.300 & 20 \\
\hline Monte Dourado - PA & 3 & 219 & 2.320 & 560 & 1.140 & 1.180 & 26 \\
\hline
\end{tabular}

Fonte: Instituto Nacional de Meteorologia - INMET (1999), resultados médios obtidos no período de 1931 a $1990 ;$ * 1 Eucalyptus grandis, 2-E. saligna, 3-E. grandis x E. urophylla, $4-$ E. cloesiana, $5-$ E. urophylla, $6-$ E. camaldulensis, $7-$ $E$. citriodora, 8-E. tereticornis, 9-E. pellita, $10-$ híbridos; $n$ (no de árvores-amostra); $P$ (precipitação pluvial média anual); PMS (precipitação média acumulada nos seis meses secos; de abril a setembro foram os seis meses secos para todos os locais, exceto para Monte Dourado-PA, cujo período seco foi de julho a dezembro, e para a Depressão Central-RS, definido de novembro a abril); $E V P$ (evaporação, tanque Classe A); $W(P-E V P$, disponibilidade potencial de água); e $T$ (temperatura média anual). 
Quadro 3. Estimativas de biomassa ${ }^{(1)}$ e de conteúdo ${ }^{(2)}$ de nitrogênio em plantios de eucalipto com diferentes idades, em diferentes regiões do Brasil

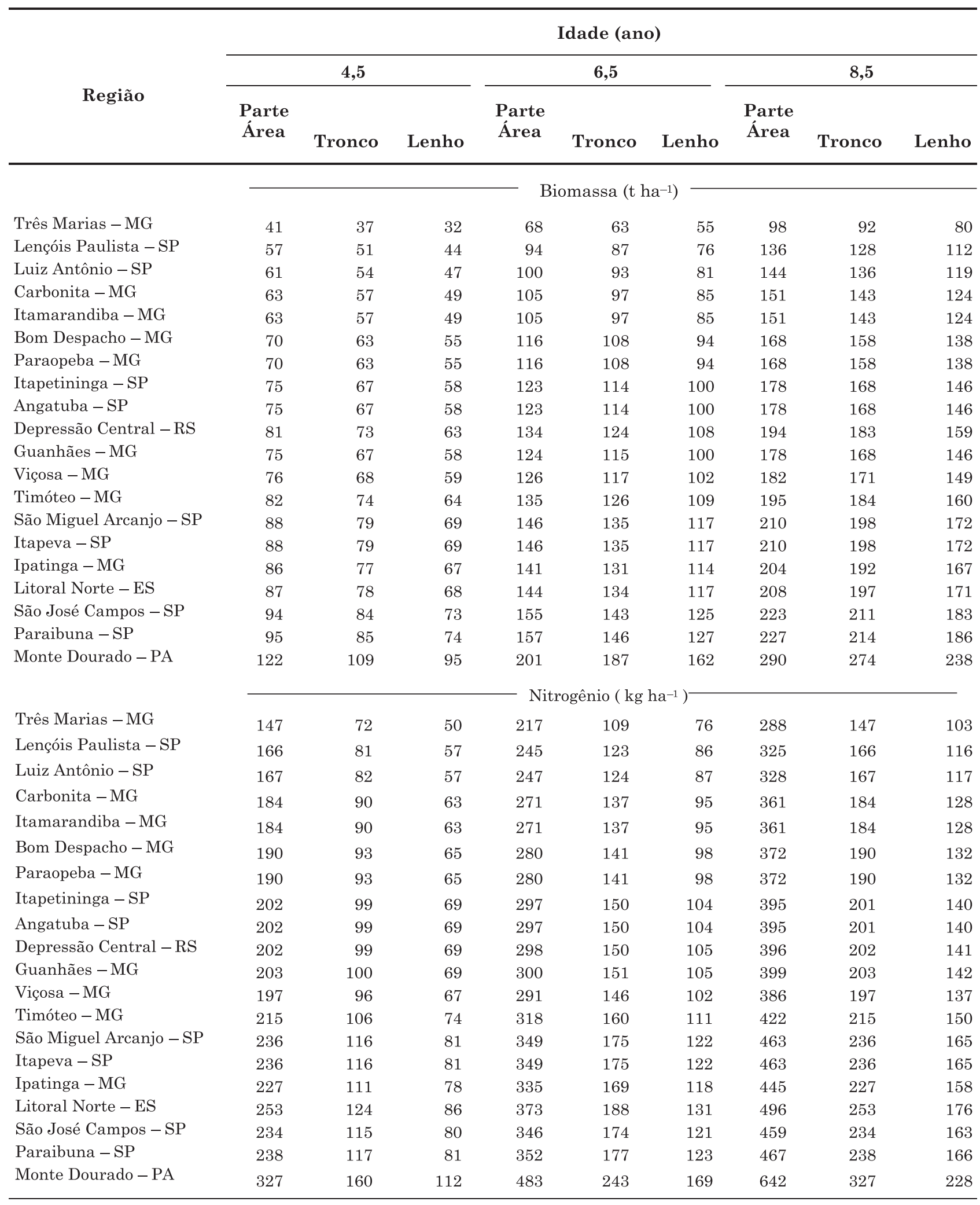

(1) $\mathrm{e}^{(2)}$ Estimativas obtidas pelo uso das equações apresentadas no quadro 2. Para biomassa, considerou-se que o lenho corresponde a $87 \%$ da biomassa de tronco; parte aérea = folha + galho + casca + lenho; e tronco $=$ casca + lenho. 
Quadro 4. Estimativas do conteúdo ${ }^{(1)}$ de fósforo e potássio em plantios de eucalipto com diferentes idades, em diferentes regiões

\begin{tabular}{|c|c|c|c|c|c|c|c|c|c|}
\hline \multirow{3}{*}{ Região } & \multicolumn{9}{|c|}{ Idade (ano) } \\
\hline & \multicolumn{3}{|c|}{4,5} & \multicolumn{3}{|c|}{6,5} & \multicolumn{3}{|c|}{8,5} \\
\hline & $\begin{array}{c}\text { Parte }^{(2)} \\
\text { Área }\end{array}$ & Tronco & Lenho & $\begin{array}{c}\text { Parte } \\
\text { Área }\end{array}$ & Tronco & Lenho & $\begin{array}{l}\text { Parte } \\
\text { Área }\end{array}$ & Tronco & Lenho \\
\hline & & & & & $\mathrm{kg} \mathrm{ha}^{-1}$ & & & & \\
\hline & & & & & Fósforo & & & & \\
\hline Três Marias - MG & 14 & 8 & 4 & 20 & 11 & 6 & 26 & 15 & 8 \\
\hline Lençóis Paulista - SP & 13 & 7 & 4 & 19 & 11 & 6 & 25 & 14 & 8 \\
\hline Luiz Antônio - SP & 12 & 6 & 3 & 17 & 10 & 5 & 23 & 13 & 7 \\
\hline Carbonita - MG & 14 & 8 & 4 & 20 & 11 & 6 & 27 & 15 & 8 \\
\hline Itamarandiba - MG & 14 & 8 & 4 & 20 & 11 & 6 & 27 & 15 & 8 \\
\hline Bom Despacho - MG & 13 & 7 & 4 & 19 & 10 & 5 & 25 & 14 & 7 \\
\hline Paraopeba - MG & 13 & 7 & 4 & 19 & 10 & 5 & 25 & 14 & 7 \\
\hline Itapetininga - SP & 17 & 9 & 5 & 25 & 14 & 7 & 32 & 18 & 10 \\
\hline Angatuba - SP & 17 & 9 & 5 & 25 & 14 & 7 & 32 & 18 & 10 \\
\hline Depressão Central - RS & 25 & 14 & 7 & 36 & 20 & 10 & 47 & 27 & 14 \\
\hline Guanhães - MG & 14 & 8 & 4 & 21 & 12 & 6 & 27 & 16 & 8 \\
\hline Viçosa - MG & 13 & 7 & 4 & 19 & 11 & 6 & 25 & 14 & 7 \\
\hline Timóteo - MG & 16 & 9 & 5 & 23 & 13 & 7 & 31 & 17 & 9 \\
\hline São Miguel Arcanjo-SP & 23 & 13 & 7 & 34 & 19 & 10 & 44 & 25 & 13 \\
\hline Itapeva - SP & 23 & 13 & 7 & 34 & 19 & 10 & 44 & 25 & 13 \\
\hline Ipatinga - MG & 17 & 9 & 5 & 25 & 14 & 7 & 32 & 18 & 10 \\
\hline Litoral Norte - ES & 22 & 12 & 6 & 32 & 18 & 9 & 42 & 24 & 12 \\
\hline São José Campos - SP & 19 & 11 & 5 & 28 & 16 & 8 & 37 & 21 & 11 \\
\hline Paraibuna - SP & 20 & 11 & 6 & 28 & 16 & 8 & 37 & 21 & 11 \\
\hline \multirow[t]{2}{*}{ Monte Dourado - PA } & 26 & 14 & 7 & 37 & 21 & 11 & 49 & 28 & 14 \\
\hline & \multicolumn{9}{|c|}{ Potássio } \\
\hline Três Marias - MG & 73 & 46 & 26 & 109 & 70 & 40 & 146 & 95 & 54 \\
\hline Lençóis Paulista - SP & 89 & 55 & 31 & 132 & 84 & 48 & 177 & 114 & 65 \\
\hline Luiz Antônio - SP & 83 & 51 & 29 & 123 & 79 & 45 & 165 & 107 & 61 \\
\hline Carbonita - MG & 94 & 58 & 33 & 140 & 89 & 51 & 188 & 121 & 69 \\
\hline Itamarandiba - MG & 94 & 58 & 33 & 140 & 89 & 51 & 188 & 121 & 69 \\
\hline Bom Despacho - MG & 93 & 58 & 33 & 139 & 88 & 50 & 186 & 120 & 68 \\
\hline Paraopeba - MG & 93 & 58 & 33 & 139 & 88 & 50 & 186 & 120 & 68 \\
\hline Itapetininga - SP & 125 & 77 & 44 & 186 & 119 & 67 & 249 & 161 & 91 \\
\hline Angatuba - SP & 125 & 77 & 44 & 186 & 119 & 67 & 249 & 161 & 91 \\
\hline Depressão Central - RS & 200 & 124 & 70 & 298 & 190 & 108 & 398 & 257 & 146 \\
\hline Guanhães - MG & 105 & 65 & 37 & 156 & 99 & 56 & 209 & 135 & 76 \\
\hline Viçosa - MG & 100 & 62 & 35 & 149 & 95 & 54 & 199 & 129 & 73 \\
\hline Timóteo - MG & 122 & 76 & 43 & 182 & 116 & 66 & 244 & 158 & 89 \\
\hline São Miguel Arcanjo-SP & 175 & 109 & 62 & 261 & 166 & 94 & 349 & 225 & 128 \\
\hline Itapeva - SP & 175 & 109 & 62 & 261 & 166 & 94 & 349 & 225 & 128 \\
\hline Ipatinga - MG & 128 & 79 & 45 & 191 & 121 & 69 & 255 & 165 & 94 \\
\hline Litoral Norte - ES & 156 & 96 & 55 & 232 & 148 & 84 & 310 & 200 & 114 \\
\hline São José Campos - SP & 156 & 96 & 55 & 232 & 148 & 84 & 310 & 200 & 114 \\
\hline Paraibuna - SP & 158 & 98 & 56 & 236 & 150 & 85 & 315 & 203 & 116 \\
\hline Monte Dourado - PA & 202 & 125 & 71 & 301 & 192 & 109 & 403 & 260 & 148 \\
\hline
\end{tabular}


Quadro 5. Estimativas do conteúdo ${ }^{(1)}$ de cálcio e magnésio em plantios de eucalipto com diferentes idades, em diferentes regiões

\begin{tabular}{|c|c|c|c|c|c|c|c|c|c|}
\hline \multirow{3}{*}{ Região } & \multicolumn{9}{|c|}{ Idade (ano) } \\
\hline & \multicolumn{3}{|c|}{4,5} & \multicolumn{3}{|c|}{6,5} & \multicolumn{3}{|c|}{8,5} \\
\hline & $\begin{array}{c}\text { Parte }^{(2)} \\
\text { Área }\end{array}$ & Tronco & Lenho & $\begin{array}{c}\text { Parte } \\
\text { Área }\end{array}$ & Tronco & Lenho & $\begin{array}{l}\text { Parte } \\
\text { Área }\end{array}$ & Tronco & Lenho \\
\hline & \multicolumn{9}{|c|}{$\mathrm{kg} \mathrm{ha}{ }^{-1}$} \\
\hline & \multicolumn{9}{|c|}{ Cálcio } \\
\hline Três Marias - MG & 84 & 60 & 17 & 132 & 97 & 27 & 185 & 138 & 39 \\
\hline Lençóis Paulista - SP & 122 & 87 & 25 & 193 & 142 & 40 & 269 & 201 & 57 \\
\hline Luiz Antônio - SP & 112 & 79 & 22 & 176 & 129 & 36 & 246 & 183 & 52 \\
\hline Carbonita - MG & 130 & 93 & 26 & 206 & 151 & 43 & 287 & 214 & 60 \\
\hline Itamarandiba - MG & 130 & 93 & 26 & 206 & 151 & 43 & 287 & 214 & 60 \\
\hline Bom Despacho - MG & 131 & 93 & 26 & 207 & 152 & 43 & 289 & 215 & 61 \\
\hline Paraopeba - MG & 131 & 93 & 26 & 207 & 152 & 43 & 289 & 215 & 61 \\
\hline Itapetininga - SP & 211 & 150 & 42 & 333 & 244 & 69 & 464 & 346 & 98 \\
\hline Angatuba - SP & 211 & 150 & 42 & 333 & 244 & 69 & 464 & 346 & 98 \\
\hline Depressão Central - RS & 489 & 347 & 98 & 771 & 565 & 160 & 1076 & 801 & 226 \\
\hline Guanhães - MG & 155 & 110 & 31 & 244 & 179 & 51 & 341 & 254 & 72 \\
\hline Viçosa - MG & 149 & 106 & 30 & 235 & 172 & 49 & 328 & 244 & 69 \\
\hline Timóteo - MG & 201 & 143 & 40 & 317 & 232 & 66 & 442 & 329 & 93 \\
\hline São Miguel Arcanjo-SP & 352 & 250 & 71 & 556 & 407 & 115 & 775 & 577 & 163 \\
\hline Itapeva - SP & 352 & 250 & 71 & 556 & 407 & 115 & 775 & 577 & 163 \\
\hline Ipatinga - MG & 211 & 150 & 42 & 333 & 244 & 69 & 465 & 346 & 98 \\
\hline Litoral Norte - ES & 269 & 192 & 54 & 425 & 312 & 88 & 593 & 442 & 125 \\
\hline São José Campos - SP & 300 & 213 & 60 & 473 & 347 & 98 & 660 & 492 & 139 \\
\hline Paraibuna - SP & 306 & 217 & 61 & 482 & 354 & 100 & 673 & 501 & 142 \\
\hline \multirow[t]{2}{*}{ Monte Dourado - PA } & 393 & 279 & 79 & 620 & 455 & 128 & 865 & 645 & 182 \\
\hline & \multicolumn{9}{|c|}{ Magnésio } \\
\hline Três Marias - MG & 20 & 12 & 4 & 29 & 18 & 6 & 39 & 24 & 8 \\
\hline Lençóis Paulista - SP & 29 & 17 & 6 & 43 & 26 & 9 & 57 & 35 & 12 \\
\hline Luiz Antônio - SP & 29 & 17 & 6 & 42 & 26 & 9 & 56 & 35 & 12 \\
\hline Carbonita - MG & 31 & 18 & 6 & 46 & 28 & 10 & 61 & 38 & 13 \\
\hline Itamarandiba - MG & 31 & 18 & 6 & 46 & 28 & 10 & 61 & 38 & 13 \\
\hline Bom Despacho - MG & 33 & 19 & 7 & 49 & 30 & 10 & 65 & 40 & 14 \\
\hline Paraopeba - MG & 33 & 19 & 7 & 49 & 30 & 10 & 65 & 40 & 14 \\
\hline Itapetininga - SP & 45 & 26 & 9 & 66 & 40 & 14 & 88 & 54 & 18 \\
\hline Angatuba - SP & 45 & 26 & 9 & 66 & 40 & 14 & 88 & 54 & 18 \\
\hline Depressão Central - RS & 78 & 46 & 16 & 116 & 70 & 24 & 154 & 95 & 32 \\
\hline Guanhães - MG & 37 & 22 & 7 & 55 & 33 & 11 & 73 & 45 & 15 \\
\hline Viçosa - MG & 37 & 22 & 7 & 55 & 33 & 11 & 73 & 45 & 15 \\
\hline Timóteo - MG & 45 & 27 & 9 & 66 & 40 & 14 & 88 & 54 & 19 \\
\hline São Miguel Arcanjo-SP & 64 & 38 & 13 & 94 & 57 & 19 & 126 & 77 & 26 \\
\hline Itapeva - SP & 64 & 38 & 13 & 94 & 57 & 19 & 126 & 77 & 26 \\
\hline Ipatinga - MG & 47 & 28 & 9 & 69 & 42 & 14 & 92 & 56 & 19 \\
\hline Litoral Norte - ES & 53 & 31 & 11 & 78 & 47 & 16 & 104 & 64 & 22 \\
\hline São José Campos - SP & 60 & 36 & 12 & 89 & 54 & 18 & 119 & 73 & 25 \\
\hline Paraibuna - SP & 61 & 36 & 12 & 91 & 55 & 19 & 121 & 74 & 25 \\
\hline Monte Dourado - PA & 74 & 44 & 15 & 110 & 66 & 23 & 146 & 90 & 31 \\
\hline
\end{tabular}

(1) Estimativas obtidas pelo uso das equações apresentadas no quadro 2; parte aérea $=$ folha + galho + casca + lenho; e tronco $=$ casca + lenho. 
As quantidades demandadas de nutrientes para produzir uma mesma biomassa em plantações de eucalipto permitem verificar se variações nas quantidades de nutrientes imobilizados na biomassa dependem mais da produção desta ou das condições de clima e solo de cada sítio. Assim, ao fixar uma mesma estimativa de biomassa da parte aérea $\left(100 t^{~ h a} a^{-1}\right)$ nas equações preditivas para as diferentes regiões, foram estimadas variações na quantidade de nutrientes nela imobilizados (Quadro 6). As maiores variações entre as quantidades de nutrientes foram estimadas para o Ca e o $\mathrm{Mg}$. Por outro lado, o $\mathrm{N}$ foi o nutriente que apresentou menor variação com um coeficiente de variação de 5,3\% entre regiões. A despeito das diferentes condições ambientais entre regiões, a estimativa de variação do conteúdo dos nutrientes na biomassa não foi ampla. Para N, P e K o coeficiente de variação foi inferior a $20 \%$ entre regiões, sendo o maior coeficiente de $38,2 \%$ para $\mathrm{Ca}$.

A pequena variação no acúmulo de nutrientes na biomassa indicaria ou pequena plasticidade do eucalipto na absorção e acúmulo de nutrientes ou baixa disponibilidade dos mesnos no solo. Esta última possibilidade é a mais provável.
A determinação de nutrientes absorvidos ao longo dos anos é importante para definir a fase de maior demanda e, eventualmente, fertilizar no período mais crítico. Observou-se que a estimativa da distribuição relativa de todos os nutrientes na parte aérea aumentou linearmente e, praticamente, não houve alteração com a idade, seguindo a mesma tendência da distribuição de biomassa (Figura 1). De acordo com as equações preditivas, aproximadamente $70 \%$ da demanda de todos os nutrientes acumula até à idade de 4,5 anos, para um ciclo de corte de 6,5 anos. Em geral, para o eucalipto, a ciclagem bioquímica torna-se mais expressiva a partir de, aproximadamente, 4,5 anos de idade, época em que a copa está totalmente desenvolvida e a ciclagem dos nutrientes contribui expressivamente para a demanda nutricional (Gonçalves et al., 2000), reduzindo, assim, a pressão sobre as reservas do solo. Segundo Miller (1995), após o estabelecimento da ciclagem bioquímica, até $66 \%$ da demanda dos nutrientes considerados móveis pode ser suprida pela retranslocação, o que reduz a probabilidade de resposta à fertilização.

Quadro 6. Estimativa do conteúdo ${ }^{(1)}$ de N, P, K, Ca e Mg na biomassa da parte aérea de eucalipto, para a produção de $100 \mathrm{t} \mathrm{ha} \mathrm{h}^{-1}$, em diferentes regiões

\begin{tabular}{|c|c|c|c|c|c|}
\hline Região & $\mathbf{N}$ & $\mathbf{P}$ & $\mathbf{K}$ & $\mathbf{C a}$ & Mg \\
\hline & & & $\operatorname{cg} 100 \mathrm{t}^{-1} \mathrm{ha}^{-}$ & & \\
\hline Três Marias - MG & 293 & 27 & 149 & 188 & 40 \\
\hline Lençóis Paulista - SP & 256 & 20 & 139 & 204 & 45 \\
\hline Luiz Antônio - SP & 247 & 17 & 123 & 176 & 42 \\
\hline Carbonita - MG & 262 & 20 & 135 & 197 & 44 \\
\hline Itamarandiba - MG & 262 & 20 & 135 & 197 & 44 \\
\hline Bom Despacho - MG & 250 & 17 & 123 & 181 & 43 \\
\hline Paraopeba - MG & 250 & 17 & 123 & 181 & 43 \\
\hline Itapetininga $-\mathrm{SP}$ & 253 & 21 & 157 & 275 & 56 \\
\hline Angatuba - SP & 253 & 21 & 157 & 275 & 56 \\
\hline Depressão Central - RS & 238 & 29 & 236 & 590 & 92 \\
\hline Guanhães - MG & 255 & 18 & 132 & 202 & 46 \\
\hline Viçosa - MG & 243 & 16 & 124 & 191 & 46 \\
\hline Timóteo - MG & 251 & 19 & 143 & 241 & 52 \\
\hline São Miguel Arcanjo - SP & 261 & 25 & 194 & 395 & 70 \\
\hline Itapeva - SP & 261 & 25 & 194 & 395 & 70 \\
\hline Ipatinga $-\mathrm{MG}$ & 256 & 19 & 145 & 243 & 53 \\
\hline Litoral Norte - ES & 281 & 24 & 173 & 304 & 58 \\
\hline São José dos Campos - SP & 246 & 20 & 164 & 319 & 63 \\
\hline Paraibuna - SP & 248 & 20 & 164 & 320 & 64 \\
\hline Monte Dourado - PA & 281 & 22 & 173 & 329 & 63 \\
\hline Amplitude & $238-293$ & $16-29$ & $123-236$ & $176-590$ & $40-92$ \\
\hline Média & 257,3 & 21,8 & 154,2 & 270,2 & 54,5 \\
\hline CV (\%) & 5,3 & 16,9 & 19,0 & 38,2 & 23,9 \\
\hline
\end{tabular}

(1) Estimativas obtidas pelo uso das equações apresentadas no quadro 2; parte aérea = folha + galho + casca + lenho. 


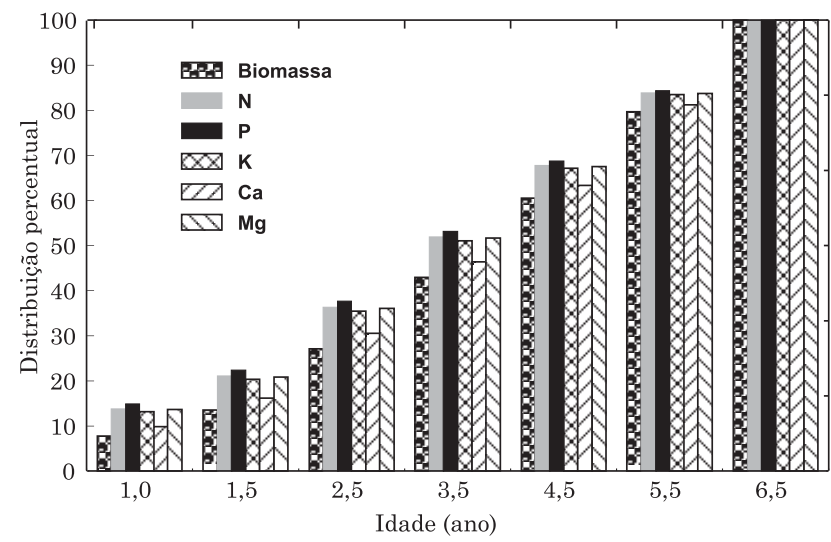

Figura 1. Distribuição relativa de biomassa e de nutrientes na parte aérea de eucalipto no Brasil, considerando a idade, assumindo 6,5 anos como idade de corte.

Segundo Novais et al. (1986), o nível crítico de manutenção refere-se ao teor do elemento que o solo deve ter, além do requerido para o crescimento e desenvolvimento inicial da muda, em condições de campo, para atingir determinada produtividade ao final do ciclo. Assim, para inferir sobre a fertilidade do solo em cada sítio, transformou-se o conteúdo de nutrientes da planta em unidade de análise de solo. Baseado nesta estimativa, pode-se inferir que a fertilidade do solo provavelmente foi mais elevada nas regiões mais produtivas (Quadro 7). Os teores de K, Ca e Mg estimados para a região de Três Marias-MG, com produtividade de $19,4 \mathrm{~m}^{3} \mathrm{ha}^{-1} \mathrm{ano}^{-1}$, apresentaram-se abaixo dos valores de níveis críticos considerados adequados para produtividade de $20 \mathrm{~m}^{3} \mathrm{ha}^{-1}$ ano $^{-1}$, para a camada de 0 - $20 \mathrm{~cm}$ de solo, num ciclo de corte de dez anos, confome sugerido por Novais et al. (1986). Este comportamento foi observado, por meio das estimativas, nas sete regiões de menor produtividade. Ao considerar apenas o $\mathrm{K}$ e o $\mathrm{Ca}$, verificou-se que 13 das 20 regiões apresentaram teores inferiores aos do nível crítico de manutenção para as produtividades obtidas. Por conseguinte, o nível crítico de manutenção, ou seja, o teor de nutrientes no solo abaixo do qual há possibilidade de média a alta resposta à fertilização de manutenção indica que os plantios de eucalipto dessas regiões, provavelmente, responderão à aplicação de tais nutrientes. A produção obtida, apesar de os teores estimados estarem abaixo dos críticos, pode ser explicada pela possível exploração radicular abaixo dos $20 \mathrm{~cm}$ de profundidade ou uma maior eficiência de utilização dos nutrientes pelas árvores nesses sítios em comparação com os relatados por Novais et al. (1986).

Quadro 7. Incremente médio anual (IMA) de Biomassa e volume do tronco de eucalipto e teores de P, K, Ca e Mg no solo necessários para atender à demanda da árvore, de acordo com a região de cultivo

\begin{tabular}{|c|c|c|c|c|c|c|}
\hline \multirow{2}{*}{ Região } & \multicolumn{2}{|c|}{ IMA $^{(1)}$} & \multicolumn{4}{|c|}{ Nutriente $^{(2)}$} \\
\hline & Biomassa & Volume & $\mathbf{P}$ & $\mathbf{K}$ & $\mathbf{C a}$ & Mg \\
\hline & t ha ${ }^{-1}$ ano $^{-1}$ & $\mathbf{m}^{3} \mathbf{h a}^{-1} \mathbf{a n o}^{-1}$ & \multicolumn{2}{|c|}{$\mathbf{m g} \mathbf{d m}^{-3}$} & \multicolumn{2}{|c|}{$\mathrm{cmol}_{\mathrm{c}} \mathbf{d m}^{-3}$} \\
\hline Três Marias - MG & 9,7 & 19,4 & 5,6 & 35 & 0,24 & 0,07 \\
\hline Lençóis Paulista - SP & 13,4 & 26,8 & 5,4 & 42 & 0,35 & 0,11 \\
\hline Luiz Antônio - SP & 14,3 & 28,6 & 4,8 & 39 & 0,32 & 0,11 \\
\hline Carbonita - MG & 14,9 & 29,8 & 5,7 & 45 & 0,38 & 0,11 \\
\hline Itamarandiba - MG & 14,9 & 29,8 & 5,7 & 45 & 0,38 & 0,11 \\
\hline Bom Despacho - MG & 16,6 & 33,2 & 5,2 & 44 & 0,38 & 0,12 \\
\hline Paraopeba - MG & 16,6 & 33,2 & 5,2 & 44 & 0,38 & 0,12 \\
\hline Itapetininga - SP & 17,5 & 35,1 & 6,9 & 59 & 0,61 & 0,16 \\
\hline Angatuba - SP & 17,5 & 35,1 & 6,9 & 59 & 0,61 & 0,16 \\
\hline Depressão Central - RS & 19,1 & 38,2 & 10,0 & 95 & 1,41 & 0,29 \\
\hline Guanhães - MG & 17,7 & 35,4 & 5,8 & 50 & 0,45 & 0,14 \\
\hline Viçosa - MG & 18,0 & 36,0 & 5,3 & 47 & 0,43 & 0,14 \\
\hline Timóteo - MG & 19,4 & 38,8 & 6,5 & 58 & 0,58 & 0,17 \\
\hline São Miguel Arcanjo - SP & 20,8 & 41,5 & 9,4 & 83 & 1,02 & 0,24 \\
\hline Itapeva - SP & 20,8 & 41,5 & 9,4 & 83 & 1,02 & 0,24 \\
\hline Ipatinga - MG & 20,2 & 40,3 & 6,9 & 61 & 0,61 & 0,17 \\
\hline Litoral Norte - ES & 20,6 & 41,2 & 8,9 & 74 & 0,78 & 0,19 \\
\hline São José dos Campos - SP & 22,0 & 44,0 & 7,8 & 74 & 0,87 & 0,22 \\
\hline Paraibuna - SP & 22,5 & 44,9 & 7,9 & 75 & 0,88 & 0,23 \\
\hline Monte Dourado - PA & 28,8 & 57,5 & 10,4 & 96 & 1,13 & 0,27 \\
\hline
\end{tabular}

(1) Valores estimados para árvores com 6,5 anos; relação matéria seca volume $=0,5$; ${ }^{(2)}$ nutrientes na biomassa do tronco, transformados em unidade de análise de solo, considerando a profundidade de solo de 0 a $20 \mathrm{~cm}$, densidade igual a 1 e uma recuperação de $100 \%$ do nutriente pelas árvores; os valores em negrito estão abaixo do nível crítico de manutenção para as diferentes produtividades sugeridos por Novais et al. (1986). 
No outro extremo, na região de Monte DouradoPA, com produtividade de $57,5 \mathrm{~m}^{3} \mathrm{ha}^{-1}$ ano $^{-1}$, os teores de nutrientes preditos apresentaram-se acima dos níveis críticos de manutenção, o que pode estar evidenciando baixa resposta à aplicação desses nutrientes. Em 16 das 20 regiões, os teores estimados para o $\mathrm{K}$ foram inferiores aos do nível crítico de manutenção. Portanto, para a manutenção de elevadas taxas de crescimento de eucalipto, especialmente em solos que suportam mais de uma rotação, deverá ser feita a fertilização potássica.

O conhecimento da alocação de nutrientes nos componentes das plantas permite ao silvicultor optar por técnicas de colheita que reduzam a exportação de nutrientes do sítio. Neste estudo, a predição da alocação diferiu entre os componentes e entre os nutrientes. $\mathrm{O}$ conteúdo de nutrientes na copa variou de 108 a 240 $\mathrm{kg} \mathrm{ha}{ }^{-1}$, para $\mathrm{N}$, de 7 a 16 , para $\mathrm{P}$, de 39 a 109 , para $\mathrm{K}$, de 35 a 206, para $\mathrm{Ca}$, e de 11 a 46 , para $\mathrm{Mg}$, considerando um ciclo de corte de 6,5 anos (Quadros de 3, 4 e 5). Em média, a alocação de N, P, K, Ca e Mg na copa foi de 50, 44, 36, 27 e $39 \%$, respectivamente, em relação à quantidade da biomassa da parte aérea (Quadro 8). Com o aumento da idade, notou-se tendência de redução dos nutrientes alocados na copa e aumento dos nutrientes alocados no tronco. Este resultado mostra a sensibilidade das equações adotadas a uma variação biológica conhecida, uma vez que com o aumento da idade a proporção de copa reduz e a de tronco aumenta.

Quadro 8. Alocação percentual média de biomassa da parte aérea e de nutrientes nos diferentes componentes da árvore em plantios de eucalipto no Brasil, de acordo com a idade do povoamento

\begin{tabular}{|c|c|c|c|c|c|}
\hline & Idade & Copa $1 \perp$ & Tronco피 & Lenho(1) $^{(1)}$ & Casca $^{(2)}$ \\
\hline & ano & \multicolumn{2}{|r|}{$-\%$} & & \\
\hline \multirow{4}{*}{ Biomassa } & 4,5 & 10 & 90 & 78 & 13 \\
\hline & 6,5 & 7 & 93 & 81 & 13 \\
\hline & 8,5 & 6 & 94 & 82 & 13 \\
\hline & 4,5 & 51 & 49 & 34 & 31 \\
\hline \multirow[t]{3}{*}{ Nitrogênio } & 6,5 & 50 & 50 & 35 & 30 \\
\hline & 8,5 & 49 & 51 & 36 & 30 \\
\hline & 4,5 & 45 & 55 & 29 & 47 \\
\hline \multirow[t]{3}{*}{ Fósforo } & 6,5 & 44 & 56 & 29 & 48 \\
\hline & 8,5 & 43 & 57 & 30 & 47 \\
\hline & 4,5 & 38 & 62 & 35 & 43 \\
\hline \multirow[t]{3}{*}{ Potássio } & 6,5 & 36 & 64 & 36 & 43 \\
\hline & 8,5 & 35 & 65 & 37 & 43 \\
\hline & 4,5 & 29 & 71 & 20 & 72 \\
\hline \multirow[t]{3}{*}{ Cálcio } & 6,5 & 27 & 73 & 21 & 72 \\
\hline & 8,5 & 26 & 74 & 21 & 72 \\
\hline & 4,5 & 41 & 59 & 20 & 66 \\
\hline \multirow[t]{2}{*}{ Magnésio } & 6,5 & 39 & 61 & 21 & 66 \\
\hline & 8,5 & 38 & 62 & 21 & 66 \\
\hline
\end{tabular}

$\overline{\text { Biomassa da parte aérea }=\text { folha }+ \text { galho }+ \text { casca }+ \text { lenho; }}$ tronco $=$ casca + lenho; e copa $=$ folha + galho .

(1) percentagem em relação à biomassa da parte aérea e ${ }^{(2)}$ percentagem em relação à biomassa de tronco.
Baseada na prognose obtida pelas equações, a biomassa da casca representa, em média, $13 \%$ da biomassa do tronco (Quadro 8). Entretanto, contém $72 \%$ de $\mathrm{Ca}, 66$ de $\mathrm{Mg}, 47$ de $\mathrm{P}, 43$ de $\mathrm{K}$ e 30 de $\mathrm{N}$ do total de nutrientes exportados do sítio pela colheita do tronco. Considerando um ciclo de corte de 6,5 anos (Quadros de 3, 4 e 5), a amplitude de variação do conteúdo de nutrientes na casca foi, em kg ha-1, de 70 a $405(\mathrm{Ca})$, de 12 a $46(\mathrm{Mg})$, de 5 a $10(\mathrm{P})$, de 30 a 83 $(\mathrm{K})$ e de 33 a $74(\mathrm{~N})$. A casca do eucalipto é importante no ciclo biogeoquímico, por ser dreno de nutrientes e por ser normalmente retirada do sítio pela colheita florestal.

Estudos em vários ecossistemas têm demonstrado expressiva redução no Ca trocável do solo, em virtude de sua absorção pelas florestas (Binkley, 1989; Federer et al.,1989). Para condições de clima tropical com solos altamente intemperizados, a exportação de nutrientes pode comprometer a produtividade futura em sítios pobres neste nutriente, se não for adotado um correto manejo florestal e da fertilidade do solo.

A imobilização de maior proporção de nutrientes na biomassa em relação à camada superficial do solo pode ser considerada um mecanismo de conservação de nutrientes em florestas tropicais. Esta imobilização pode evitar perda por erosão e lixiviação (Cuevas \& Lugo, 1998) e contribui para aumentar o teor de nutrientes das suas camadas superficiais, visto que o sistema radicular do eucalipto explora camadas profundas do seu perfil e pode realocar os nutrientes para as camadas superficiais. A intensidade da colheita florestal adotada determina a quantidade de nutrientes exportados do sítio. Admitindo um ciclo de 6,5 anos, pode-se inferir, por meio das estimativas realizadas, que no tronco estão contidos $50 \%$ de N, 56 de P, 64 de K, 73 de Ca e 61 de $\mathrm{Mg}$ da biomassa aérea. Estes percentuais podem ser sensivelmente reduzidos se ocorrer o descascamento no campo. A colheita apenas do lenho é ecologicamente mais correta para as condições tropicais de solos altamente intemperizados; quanto mais pobre for o solo, mais relevante será a permanência da casca, o que contribuirá para manter a qualidade e a capacidade produtiva dos solos.

\section{CONCLUSÕES}

1. A proporção de biomassa da copa reduziu com a idade e a do tronco e do lenho aumentou.

2. A proporção média de biomassa da casca em relação à biomassa da parte aérea foi de $10 \%$ e em relação à biomassa do tronco, de $13 \%$.

3. A maior proporção de todos os nutrientes (de 63 a $69 \%$ ) na biomassa acumulou-se até à idade de 4,5 anos, quando foi considerado um ciclo de corte de 6,5 anos. 
4. Aproximadamente, $75 \%$ de N, P, K, Ca e Mg estão em outros componentes da parte aérea da árvore, e não no lenho, evidenciando a importância do descascamento e manutenção da copa no campo.

\section{AGRADECIMENTOS}

À Fundação Coordenação de Aperfeiçoamento de Pessoal de Nível Superior (CAPES), pela concessão da bolsa de estudos para realização deste trabalho.

À Universidade da Flórida (UFL), em particular ao Departamento de Solos e da Água, por possibilitar a realização deste trabalho na referida universidade.

Ao Programa de Pesquisa em Solos e Nutrição de Eucalipto do Departamento de Solos da Universidade Federal de Viçosa, pela cessão dos dados utilizados neste trabalho.

\section{LITERATURA CITADA}

BARROS, N.F. \& COMERFORD, N.B. Sustentabilidade da produção de florestas plantadas na região tropical. In: ALVAREZ V., V.H.; SCHAEFER, C.E.G.R.; BARROS, N.F.; MELLO, J.W.V. \& COSTA, L.M., eds. Tópicos em ciência do solo. Viçosa, MG, Sociedade Brasileira de Ciência do Solo, 2002. v.2. p.487-592.

BINKLEY, D. Contributing to 20-yr decrease in soil $\mathrm{pH}$ in an old-field plantation of lobolly pine. Biogeochemistry, v.8, p.39-54, 1989.

CUEVAS, E. \& LUGO, A.E. Dynamics of organic matter and nutrient return from litterfall in stands of ten tropical tree plantation species. For. Ecol. Manag., 112:263-279, 1998.

EPSTEIN, E. \& BLOOM, A.J. Nutrição mineral de plantas: Princípios e perspectivas. Londrina, Planta, 2006. 402p.

FEDERER, C.A.; HORNBECK, J.W.; TRITTON, L.M.; MARTIN, C.W.; PIERCE, R.S. \& SMITH, C.T. Long-term depletion of calcium and other nutrients in Eastern U.S. forests. Environ. Manag., 13:593-601, 1989.

GONÇALVES, J.L.M.; STAPE, J.L.; BENEDETTI, V.; FESSEL, V.A.G. \& GAVA, J.L. Reflexos do cultivo mínimo e intensivo do solo em sua fertilidade e na nutrição das árvores. In: GONÇALVES, J.L.M. \& BENEDETTI, V., eds. Nutrição e fertilização florestal. Piracicaba, IPEF, 2000. p.1-49.

INSTITITUTO NACIONAL DE METEOROLOGIA - INMET. Disponível em: <http://www.inmet.br 1999>. Acesso em: 15 de mar. de 1999.
LINDER, S.; MCMURTRIE, R.E. \& LANDSBERG, J.J. Global change impacts on managed forests. In: WALKER, B. \& STEFFEN, W., eds. Global change and terrestrial ecosystems. Cambridge, Cambridge University Press, 1996. p.275-290. (IGBP Book Series, 2)

MILLER, H.G. The influence of stand development on nutrient demand, growth and allocation. Plant Soil, 168-169:225232, 1995 .

NOVAIS, R.F. \& BARROS, N.F. Sustainable agriculture and forestry production systems on acid soils: Phosphorus as a case-study. In: MONIZ, A.C.; FURLANI, A.M.C.; SCHAFFERT, R.E.; FAGERIA, N.K.; ROSOLEM, C.A. \& CANTARELLA, H., eds. Plant-soil interactions as low $\mathrm{pH}$ : Sustainable agriculture and forestry production. Viçosa, MG, Brazilian Soil Science Society, 1997. p.39-51.

NOVAIS, R.F.; BARROS, N.F. \& NEVES, J.C.L. Interpretação de análise química do solo para o crescimento de Eucalyptus spp. - níveis críticos de implantação e de manutenção. R. Árvore, 10:105-111, 1986.

PEREIRA, J.S.; MADEIRA, M.V.; LINDER, S.; ERICSSON, T.; TOMEÂ, M. \& ARAUÂ J.M.C. Biomass production with optimised nutrition in Eucalyptus globulus plantations. In: PERIERA, J.S. \& PEREIRA, H., eds. Eucalyptus for biomass production. Brussells, Commission of the European Communities, 1994. p.13-30.

RAISON, R.J.; MYERS, B.J. \& BENSON, M.L. Dynamics of Pinus radiata foliage in relation to water and nitrogen stress I. Needle production and properties. For. Ecol. Manag., 2:139-158, 1992.

SANTANA, R.C. Predição de biomassa e alocação de nutrientes em povoamentos de eucalipto no Brasil. Viçosa, MG, Universidade Federal de Viçosa, 2000. 56p.(Tese de Doutorado)

SANTANA, R.C.; BARROS, N.F. \& NEVES, J.C.L. Eficiência nutricional e sustentabilidade da produção em procedências de Eucalyptus grandis e Eucalyptus saligna em sítios florestais do Estado de São Paulo. R. Árvore, 26:447-457, 2002.

SANTANA, R.C.; BARROS, N.F. \& NEVES, J.C.L. Produção de biomassa e conteúdo de nutrientes de procedências de Eucalyptus grandis e Eucalyptus saligna em alguns sítios florestais do Estado de São Paulo. Sci. For., 56:155-169, 1999.

STAPE, J.L.; BINKLEY, D.; JACOB, W.S. \& TAKAHASHI, E.N. A twin-plot approach to determine nutrient limitation and potential productivity in eucalyptus plantations at landscape scales in Brazil. For. Ecol. Manag., 223:358362,2006 .

TURNER, J.; KNOTT, J. \& LAMBERT, M.J. Fertilization of Pinus radiata after thinning: I. Productivity gains. Austr. For., 59:7-12, 1996. 
